\title{
Vertical Takeoff and Landing (VTOL) Small Unmanned Aircraft Systems (sUAS)
}

\author{
Bettina Mrusek* \\ College of Aeronautics, Embry-Riddle Aeronautical University-Worldwide, USA
}

Submission: January 12, 2021; Published: March 10, 2021

*Corresponding author: Bettina Mrusek, College of Aeronautics, Embry-Riddle Aeronautical University-Worldwide, USAia

\section{Abstract}

Technology has paved the way for a variety of applications for unmanned aircraft systems. Vertical takeoff and landing (VTOL) small, unmanned aircraft systems (sUAS). VTOL sUAS aircraft are capable of vertical operation and include rotary, fixed-wing, and hybrid systems which incorporate both rotary and fixed-wing characteristics. There are many advantages to these aircraft, given their size and maneuverability. They are rapidly deployable, do not require the use of a runway, and are capable of translation flight (slow, fast, lateral, etc.). From an industry perspective, there are many applications and uses for these aircraft. In the agriculture industry, these aircraft can be used for aerial mapping and digital imaging. They are also useful in the construction of bridges or buildings, as they can travel to locations which are difficult or unsafe for humans. However, in today's aviation industry, there is a significant emphasis placed on sustainable aviation. Those aircraft that do not fall into this category may not reap the full benefits of today's aviation market. While traditionally, VTOL small, unmanned aircraft were not fuel efficient, advances in technology have created many opportunities for this platform, including electric motors and ultimately, enhanced fuel efficiency.

Keywords: Vertical takeoff and landing; Small unmanned aircraft systems; Aviation sustainability

\section{Vertical Takeoff and Landing Small Unmanned \\ Aircraft Systems}

There are many types of unmanned aircraft platforms and each has its own unique purpose and applicable mission sets (Austin, 2010). Vertical takeoff and landing (VTOL) small, unmanned aircraft systems (sUAS) platforms, for example, have many practical industry applications [1,2]. In the agriculture industry, these aircraft can be used for aerial mapping and digital imaging. They are also useful in the construction of bridges or buildings, as they can travel to locations which are difficult or unsafe for humans. They are unique, given that they can achieve lift without the requirement of a runway. To understand the full range of applications and future uses for these aircraft, the evolution of VTOL unmanned aircraft, as well as a review of the operational characteristics common to these aircraft will be explored. Current uses for these aircraft will also be included in the evaluation, from both recreational and commercial perspectives.

There are many clear advantages to this aircraft platform, such as their ability to rapidly deploy and translational capabilities. However, they are limited by their fuel efficiency and reduced range $[3,4]$. While these limitations may seem insignificant, considering the emphasis placed on aviation and aircraft sustainability [5], the fuel consumption rates of this platform may hinder its potential for future growth. This paper will review the VTOL platform, while examining its strengths and weaknesses to determine the extent of the fuel efficiency problem.

\section{History}

Before discussing the evolution of a specific sUAS platform, it is necessary to first understand how and when these aircraft were first used. While the Wright brothers introduced the world to manned aviation, using a powered aircraft [6], the first report of an unmanned aircraft was during the Battle of Fleurus in 1794 when French forces used a balloon to observe the battlefield [4]. The benefits of such a system were soon realized and the applications of these aircraft grew. By the 1990's, the use of unmanned aircraft by military forces had grown tremendously. They were no longer simply observation systems, they were fitted with cargo, torpedos, and flares and were incredibly beneficial in battle.

As the technology of UAS grew, the shift from tactical to practical use became evident. These systems were smaller and more manageable than ever before, which paved the way for recreational and commercial use [4]. As such, several platforms of unmanned aircraft arose, such as fixed-wing, rotary-wing, and 
VTOL. While this list is by no means inclusive, they do represent three of the most common commercial off the shelf types.

With an understanding of how unmanned aircraft evolved, a deeper understanding of a single platform type, VTOL, will be discussed. Although the focus of the paper is on small VTOL unmanned aircraft, both small and those larger than 55 pounds will be presented. The introduction of VTOL unmanned aircraft dates to the early 1960's and progressed into the 1970's and 80 's as the technology of these systems became more advanced [5]. Figure 1 depicts the number of unmanned VTOL commercial platforms developed between 1960 and 2012.

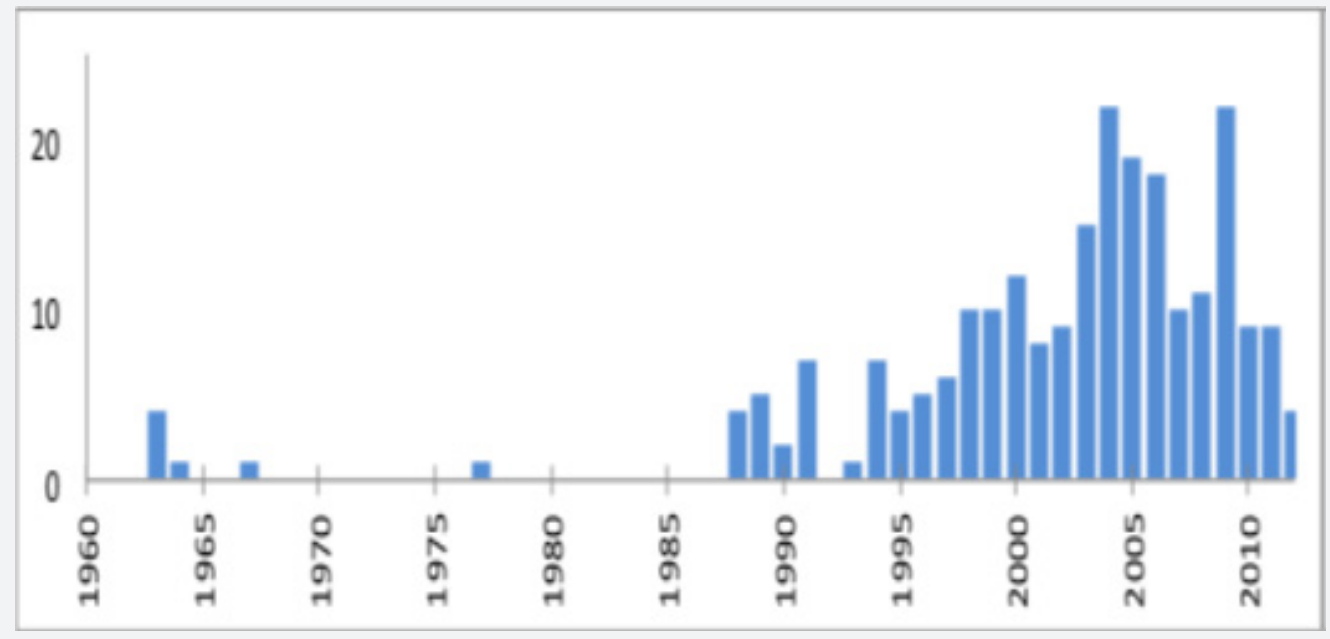

Figure 1: Unmanned VTOL commercial platform systems that were developed between 1960 and 2012 [7].

\section{Operational Characteristics}

In this next section, the different operational characteristics of VTOL aircraft will be explored. It is important to note that the design of VTOL aircraft can vary widely. Some systems include rotary-wing mechanisms, while others are fixed-wing type designs that are capable of vertical operations. Hybrid systems are also prevalent, which include both rotary and fixed-wing aspects (Figure 2).

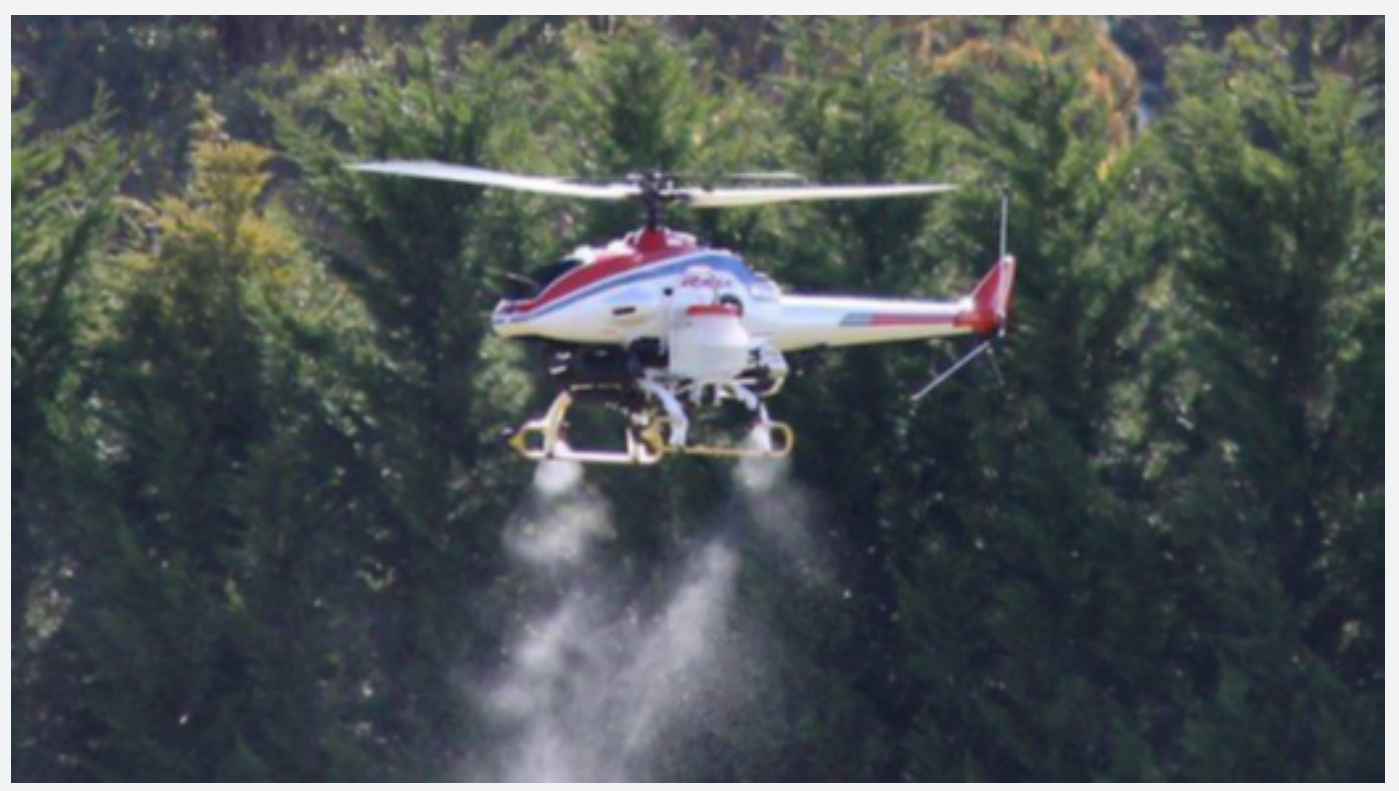

Figure 2: Yamaha RMAX VTOL aircraft applying pesticide/herbicide (Yamaha Motor Australia, n.d.). 


\section{Airframe}

While the airframe for a VTOL unmanned aircraft may exhibit similar characteristics of other fixed-wing or rotary-wing aircraft, the difference lies in its ability to generate thrust without the need for a runway $[4,7]$. Configurations for the airframe consist of single or multiple propellers, which are strategically placed in order to achieve the desired lift [8]. The placement of the propellers depends on the intent for the aircraft. For example, the below image depicts a Yamaha RMAX VTOL aircraft. The design is like modern helicopters, where a single, large propeller is used to generate lift. This type of aircraft can be used in precision agriculture to apply pesticides or herbicides. In addition to single propeller designs, VTOL airframes can also include quad-copter configurations. The use of four (or more) propellers allows the aircraft to achieve lift similarly to the single propeller but allow for enhanced mobility [9]. The below image is an Aeryon Scout VTOL aircraft.

\section{Powerplant Configurations}

The airframe design and powerplant configuration of an aircraft is ultimately what allows it to achieve and sustain flight. The powerplant is the mechanism that is used to attain flight, which is, again, supported by the airframe design. While small unmanned powerplant versions are quite different than the engines of manned aircraft jets, the intent remains the same. It is a source of power which allows the aircraft to fly. However, VTOL aircraft are unique since they must achieve vertical lift using propellers and a powerplant source [10]. This is in stark contrast to aircraft that use a runway to assist in the generation of thrust. These propellers support the upward motion of the aircraft via electric or internal combustion methods. Many sUAS VTOL aircraft utilize electric energy sources given that they are often much lighter than traditional combustion mechanisms. Larger VTOL UAS, however, may use smaller internal combustion engines. Common propeller and powerplant configurations for VTOL aircraft include collective rotor, variable-pitch propellers, and fixed-pitch propellers [4]. While all three work according to the same basic principle of powerplant mechanics, some allow for a change in the pitch of the propeller controls, as with the variable-pitch. The fixed pitch, however, is the simplest of the three. It maintains a specific, controlled rotation, normally in line with manufacturer's specifications. The type of powerplant rotor used is determined by the type of VTOL aircraft (Figure 3).

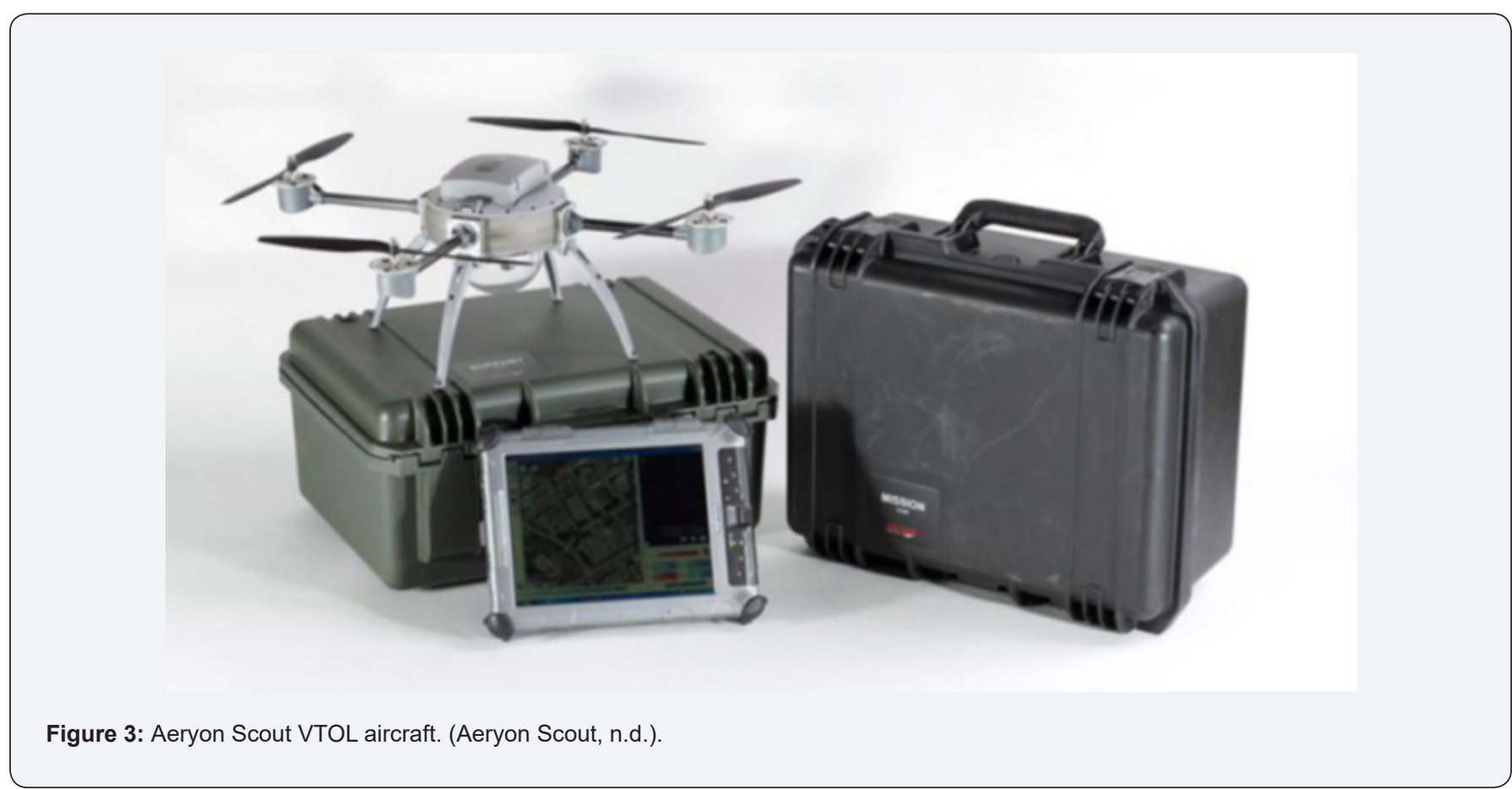

\section{Sensors}

There are many sensor packages that are available for VTOL unmanned aircraft platforms. When these aircraft first emerged in the marketplace, they were limited in terms of payload, meaning that only a few sensors could be used [7]. Today, however, advances in technology have resulted with small, lightweight sensors that can perform various functions, many of which are compatible for VTOL aircraft. For example, Precision Hawk, a leading manufacturer for small, unmanned aircraft, has an array of sensor packages to complement any aircraft platform, including VTOL. Hyperspectral, video, visual, thermal, multispectral, and LiDAR are just some of the sensors available today [2]. 


\section{Ground Support Equipment}

In addition to sensors, another important consideration is the equipment needed to operate the aircraft. Some may require multiple personnel and/or equipment. Advances in technology have made this process much smoother for unmanned aircraft, compared to their manned counterparts. VTOL small, unmanned aircraft typically require an operator, mobile device or computer, and the aircraft itself [4]. Because of the size of the aircraft, many can be easily, and safety flown by a single user. A main consideration in this process is the ability to maintain visual line of sight, which is required by the Federal Aviation Administration (FAA) under Title 14, Part 107 [11]. The low altitude limitations of this aircraft make maintaining line of sight easier than other, higher altitude aircraft.

\section{Operations, Missions, and Current Uses}

VTOL unmanned aircraft have proven especially useful in several industries including precision agriculture, emergency response, and infrastructure. Each of these industries presents a specific demand and need for VTOL aircraft. In precision agriculture, for example, these aircraft can be used to gather information related to crop health and vegetation saturation levels [2]. Emergency response personnel may need to conduct an aerial assessment of an accident to best allocate resources; VTOL aircraft can be maneuvered quickly and efficiently to provide realtime data to responders [2]. Finally, workers in the infrastructure industry may need to conduct an aerial view of a potential project or gain access to a hard to reach or even hazardous environment. VTOL aircraft have the capabilities to safely enter these areas, providing crew members with critical information [12]. The applications for these aircraft are endless.

\section{Strengths and Weaknesses}

This section will bring relevance to the previous sections by reviewing VTOL sUAS strengths and weaknesses. While there are many applications for these aircraft, it is important to distinguish its advantages and disadvantages. Some designs are more appropriate than others, depending on the use.

\section{Strengths}

The strengths of a particular aircraft are an important consideration for any pilot or operator. The practical use of the aircraft itself lie in its specific capabilities. For VTOL aircraft, advantages include a smaller launch and recovery area, its rapid deployability, and its translational capabilities. Each of these abilities make this aircraft an optimal choice for certain industries, such as precision agriculture, emergency response, and transportation. For example, the ability to launch an aircraft vertically, and without the requirement of runway, make these aircraft incredibly attractive for farmers. It allows them to gather aerial images without the burden of launching a manned aircraft, which often require a runway. For those that work in emergency response, the ability to rapidly deploy an aircraft is essential. VTOL aircraft easily fit this need. They can be rapidly deployed to dangerous areas that may be difficult for humans to reach. The final noted advantage to VTOL aircraft lies in their ability to achieve translational flight. They can easily move fast, slow, longitudinal, and laterally [2]. VTOL aircraft are also equipped to hover when needed. This is especially important for those that work in the transportation and infrastructure industries. They can fly VTOL aircraft into spaces that are dangerous or hazardous to workers. The use of certain sensors, such as video cameras allows workers to examine certain areas without having to enter them.

\section{Weaknesses}

Just as the strengths of an aircraft design are contemplated, the same can be said for the consideration of weaknesses or limitations. Depending on the intent for use of an aircraft, some weaknesses may be worthwhile. Others, however, may not. Disadvantages of the VTOL aircraft platform include fuel efficiency, airspeed limitations, and lower operational altitude capabilities. Given the recent emphasis on aviation and aircraft sustainability $[5,13]$, fuel efficiency rates are a critical consideration for not only VTOL aircraft, but all platforms. Given the unique power requirements of VTOL aircraft, fuel efficiency rates are not quite as good as their traditional fixed or rotary-wing counterparts [3]. However, for small UASs, those that are less than 55 pounds, the problem is not as significant. For larger VTOL aircraft that employ turbofan engines, fuel efficiency can be a challenge.

In addition to fuel efficiency challenges, VTOL aircraft also fly slower and at lower altitudes than other platforms. This is partly due to the design of the aircraft and its ability to achieve vertical takeoff. However, these disadvantages are often outweighed by the lack of a runway. Speed or high latitudes may not be a high a priority as vertical takeoff. In precision agriculture, for example, lower speeds and altitudes are often needed in order to obtain aerial images.

\section{Future Uses and Conclusion}

The future of VTOL unmanned aircraft is largely fueled by innovative technologies. Advances in robotics, remote sensing, and aviation sustainability have directly contributed to improvements in the design and function of these aircraft. What were once viewed as limitations are now technological breakthroughs. For example, 3W International and A-techSYN, an unmanned aircraft manufacturer, recently developed an electric engine for a VTOL aircraft. The new engine will increase range and payload, while consuming much less energy [14]. Therefore, while there are distinct disadvantages to the VTOL platform, notably in the application of aviation and aircraft sustainability, researchers and engineers are working to address these shortfalls. As these key 
areas within the aviation industry (robotics, remote sensing, and aviation sustainability) continue to advance, the resulting impact on the segment of unmanned systems will undoubtedly reap the benefits. Overall, the VTOL platform provides unique opportunities for several industries through its airframe and powerplant design, which in turn are complemented by its available sensor packages. The true benefits of this aircraft platform are evident. How it will evolve over the next decade is yet to be seen, but future projections appear bright [15-17].

\section{References}

1. Boeing (2018) Unlocking the future of flying. Innovation, Technology.

2. Precision Hawk (2018) Collect data from a wide spectrum.

3. Finger D (2016) Comparative Performance and Benefit Assessment of VTOL and CTOL UAVs.

4. Terwilliger B, Ison DC, Robbins J, Vincenzi DA (2017) Small unmanned aircraft systems guide: Exploring designs, operations, regulations, and economics. Newcastle, Washington: Aviation Supplies \& Academics, Inc.

5. Kivits R, Charles MB, Ryan N (2010) A post-carbon aviation future: Airports and the transition to a cleaner aviation sector. Futures 42(3): 199-211.

6. Wright Brothers (2008) In Philip's encyclopedia. London, UK: Philip's.

7. Watts AC, Ambrosia VG, Hinkley EA (2012) Unmanned aircraft systems in remote sensing and scientific research: Classification and considerations of use. Remote Sensing 4(6): 1671-1692.

8. Plummer L (2017) What is VTOL? A beginner's guide to vertical takeoff and landing technology. Wired online, Technology.

9. Krishnakumar R, Rasheed AM, Kumar KS (2015) Enhanced hover control of quad tilt frame UAV under windy conditions. International Journal of Advanced Robotic Systems 12(10): 146.

10. Czyba R, Szafranski G (2013) Control structure impact on the flying performance of the multi-rotor VTOL platform - design, analysis and experimental validation. International Journal of Advanced Robotic Systems 10(1): 62 .

11. (2016) Federal Aviation Administration. 14 CFR $§ 107$.

12. Rawat K, Lawrence E (2014) A mini-UAV VTOL platform for surveying applications. IAES International Journal of Robotics and Automation 3(4): 259-267.

13. Kantenbacher J, Hanna P, Cohen S, Miller G, Scarles C (2018) Public attitudes about climate policy options for aviation. Environmental Science and Policy 81: 46-53.

14. Rees M (2018) New VTOL UAS engine provides enhanced performance $\&$ fuel efficiency. International $\mathrm{GmbH}$ news.

15. Aeryon Scout VTOL aircraft (Aeryon Scout, n.d.).

16. Austin (2010) Unmanned aircraft systems UAVS design, development, and deployment. John Wiley \& Sons Ltd, West Sussex, United Kingdom.

17. Yamaha Motor Australia (n.d). Visual image. Yamaha RMAX VTOL aircraft.

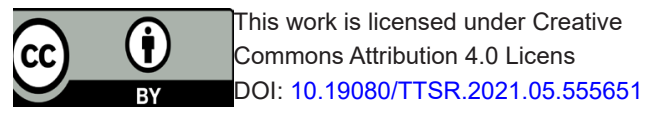

